\title{
Lowering the alcohol content of red wine does not alter its cardioprotective properties
}

\author{
Kim Lamont, Dee Blackhurst, Zulfah Albertyn, David Marais, Sandrine Lecour
}

Background. Many epidemiological, clinical and laboratory studies suggest that chronic and moderate consumption of red wine benefits cardiovascular health, because of the alcoholic content or the polyphenols/flavonoids.

Aims. The antioxidant and cardioprotective properties of a French red wine (cabernet sauvignon, 12\% alcohol by volume) were compared with those of the same wine subjected to reverse osmosis for partial removal of alcohol ( $6 \%$ alcohol by volume).

Methods. Antioxidant capacity was assessed in vitro using the oxygen radical absorbance capacity (ORAC) assay. To test the cardioprotective effect of $12 \%$ v. $6 \%$ wine, the drinking water of rats used for controls was supplemented with red wine ( $12 \%$ or $6 \%)$. After 10 days, hearts were isolated on a Langendorff system and subjected to 30 minutes of global ischaemia plus 30 minutes of reperfusion (I/R).
Results. No differences in antioxidant capacity were observed between wine of $12 \%$ and $6 \%$ alcohol content ( $n=8$ per group). Control hearts subjected to I/R presented a rate pressure product (heart rate $\mathrm{x}$ left ventricular developed pressure, expressed as a percentage of baseline value) of $16 \pm 4 \%$ (mean \pm standard error). Pretreatment with wine $12 \%$ or $6 \%$ improved the rate pressure product to $40 \pm 6 \%$ and $43 \pm 6 \%$, respectively ( $p<0.05 \mathrm{v}$. control).

Conclusion. Our findings suggest that the reduction of alcohol content from $12 \%$ to $6 \%$ in wine did not alter its antioxidant and cardioprotective properties. Moderate and regular consumption of lower alcohol content wines may confer beneficial effects without the risks associated with traditional wines of higher alcohol content.

S Afr Med J 2012;102(6):565-567.
Experimental studies suggest that red wine has cardiovascular protective effects 'beyond the alcohol'. Epidemiological, clinical and laboratory studies provide evidence for benefit from moderate alcohol consumption in cardiovascular health. ${ }^{1}$ Alcohol consumption, from whatever source, appears to have a J-shaped curve, whereby a modest intake is beneficial and either no intake or increased intake is harmful. ${ }^{2}$ The J-shaped mortality curve might in part be explained by less myocardial infarction ${ }^{3}$ and fewer incidences of heart failure. ${ }^{4}$ The maximal protective effect occurs between half and one drink per day. ${ }^{5}$ In contrast, the risk of cancers tends to increase with the amount of ethanol consumed, and it is not clear whether a threshold exists below which this side-effect is not observed. ${ }^{6}$ Therefore, the consumption of alcohol should be kept low to achieve cardioprotective benefits without enhancing the alcoholrelated side-effects on the liver.

Red wine polyphenol extracts have cardioprotective properties against ischaemia/reperfusion ${ }^{7}$ and these effects have mainly been attributed to the polyphenol resveratrol - a potent antioxidant and activator of prosurvival pathways. ${ }^{8}$ However, the proportion of the cardioprotective effect attributable to the alcohol or the polyphenols in red wine is still unclear.

We aimed to compare the antioxidant and cardioprotective properties of a French red wine (cabernet sauvignon, $12 \%$ alcohol by

Hatter Institute for Cardiovascular Research in Africa, Department of Medicine, University of Cape Town

Kim Lamont, MSc

Zulfah Albertyn, MSc

Sandrine Lecour, PharmD, $\mathrm{PhD}$

Division of Chemical Pathology, Clinical Laboratory Sciences, University of Cape Town

Dee Blackhurst, $\mathrm{PhD}$

David Marais, MB ChB, FCP volume: wine 12\%) with the same wine treated for partial removal of alcohol content ( $6 \%$ alcohol by volume: wine $6 \%)$.

\section{Methods}

French red, rosé and white wines were used in this study. To achieve their corresponding low-alcohol product, alcohol was removed by the lirisation process ${ }^{9}$ from $12 \%$ to $6 \%$ alcohol by volume, without any other alteration of the original composition (http://www. michaelpaetzold.com). ${ }^{9}$

The antioxidant properties of the wines were evaluated in vitro using the oxygen radical absorbance capacity (ORAC) assay with fluorescein. ${ }^{10}$ Values were measured as $\mathrm{mmol} / \mathrm{l}$ Trolox equivalents (TE).

The drinking water used for the male Long Evans control rats (CTL) group was supplemented by red wine (French cabernet sauvignon: wine $12 \%$ group), with its corresponding low-alcohol product (wine $6 \%$ group) or with the alcohol extracted from the wine ( $6 \%$ or $12 \%$ volume/volume). The different drinking solutions were prepared daily by adding one part of red wine or alcohol solution to seven parts of drinking water (equivalent of 2 glasses per day relative to body weight). ${ }^{11}$ After 10 days of treatment, rats were anaesthetised with sodium pentobarbitone $(60 \mathrm{mg} / \mathrm{kg}$, intraperitoneal) and received heparin (200 IU intravenously (IV)).

Hearts were excised and perfused on a Langendorff perfusion system. ${ }^{12}$ Cardiac parameters were monitored continuously and included heart rate (HR), left ventricular developed pressure (LVDP; the difference between left ventricular end systolic pressure and left ventricular end diastolic pressure) and the coronary flow. Rate pressure product (RPP) was calculated as the HR $\mathrm{x}$ LVDP for a specific time point. All rat hearts were equilibrated for 30 minutes and subjected to 30 minutes of global ischaemia $\left(37^{\circ} \mathrm{C}\right)$ followed by 30 minutes of reperfusion (I/R). The study was approved by the Animal Research Ethic Committee, University of Cape Town.

Data were expressed as mean values \pm standard error of the mean and were analysed by performing multiple group comparisons using one-way analysis of variance (ANOVA) followed by Tukey post hoc test (Graph Pad Instat). A value of $p<0.05$ was considered to be statistically significant. 


\section{Results}

The in vitro antioxidant properties of red wine were much higher than those of rosé or white wine $(30.6 \pm 0.9,6.3 \pm 0.1$ and $4.0 \pm 0.1$ $\mathrm{mmol} / 1$ Trolox equivalent, respectively, $p<0.05 \mathrm{v}$. white wine) (Fig. $1)$. These results conform to previous reports. The reduction of the alcohol content to $6 \%$ in the 3 types of wine did not affect the antioxidant properties of the wine $(30.6 \pm 0.9 \mathrm{mmol} / \mathrm{l}$ Trolox equivalent for the red wine $12 \%$ and $30.4 \pm 0.9$ for the wine $6 \%$ (not significant (NS); $n=6$ per group).

Pretreatment with wine (12\% or $6 \%$ ) or alcohol (12\% or $6 \%)$ did not affect the functional parameters of the isolated rat heart prior to the ischaemic insult. After I/R, large alterations of haemodynamic function were observed in all groups. For the ischaemic control group, the end of the reperfusion was marked by a dramatic decrease of LVDP, HR and coronary flow compared with the pre-ischaemic values (Table 1).

Pretreatment with wine $12 \%$ or $6 \%$ improved LVDP function after reperfusion (Table 1) compared with control hearts $(p<0.05)$. No difference was observed in the haemodynamic parameters between wine $12 \%$ and wine $6 \%$. Control hearts subjected to $\mathrm{I} / \mathrm{R}$ presented a RPP (expressed as a percentage of baseline value) of $19 \pm 1 \%$ (Fig. 2). Pretreatment with wine $12 \%$ or $6 \%$ improved the RPP to $37 \pm 5 \%$ and $37 \pm 4 \%$, respectively ( $p<0.05$ v. control). Pretreatment with alcohol $12 \%$ or $6 \%$ for 10 days did not improve the LVDP, HR and coronary flow after 30 minutes of reperfusion compared with control hearts (Table 1; NS). Similarly, the RPP did not improve with pretreatment of alcohol $12 \%$ or $6 \%$ compared with the control group (Fig. 2, NS).

\section{Discussion}

Our data provide the first evidence that chronic and moderate administration of red wine to rats for 10 days is protective against an $I / R$ insult. Most importantly, we show that the reduction of

Table 1. Haemodynamic parameters of perfused rat hearts

\begin{tabular}{|c|c|c|c|}
\hline & Pre-ischaemic & $\begin{array}{l}\text { Reperfusion } \\
5 \mathrm{~min}\end{array}$ & $\begin{array}{l}\text { Reperfusion } \\
30 \mathrm{~min}\end{array}$ \\
\hline \multicolumn{4}{|l|}{ LVDP (mmHg) } \\
\hline Control & $87 \pm 1$ & $16 \pm 2$ & $23 \pm 3$ \\
\hline Wine $6 \%$ & $87 \pm 2$ & $27 \pm 5^{*}$ & $36 \pm 3^{*}$ \\
\hline Wine $12 \%$ & $88 \pm 3$ & $26 \pm 3^{*}$ & $38 \pm 3^{*}$ \\
\hline Alcohol 6\% & $87 \pm 5$ & $12 \pm 2$ & $26 \pm 2$ \\
\hline Alcohol 12\% & $87 \pm 6$ & $10 \pm 4$ & $17 \pm 2$ \\
\hline \multicolumn{4}{|c|}{ Heart rate (bts/min) } \\
\hline Control & $340 \pm 17$ & $192 \pm 32$ & $227 \pm 20$ \\
\hline Wine $6 \%$ & $307 \pm 13$ & $260 \pm 34$ & $270 \pm 18$ \\
\hline Wine $12 \%$ & $300 \pm 9$ & $250 \pm 37$ & $253 \pm 29$ \\
\hline Alcohol 6\% & $304 \pm 10$ & $228 \pm 27$ & $260 \pm 29$ \\
\hline Alcohol 12\% & $328 \pm 15$ & $232 \pm 66$ & $286 \pm 36$ \\
\hline \multicolumn{4}{|l|}{ Flow $(\mathrm{ml} / \mathrm{min})$} \\
\hline Control & $13.0 \pm 1.1$ & $7.3 \pm 0.4$ & $5.5 \pm 0.3$ \\
\hline Wine $6 \%$ & $13.5 \pm 1.0$ & $9.6 \pm 0.7$ & $5.6 \pm 0.2$ \\
\hline Wine $12 \%$ & $11.8 \pm 1.4$ & $10.0 \pm 0.9$ & $6.7 \pm 0.4$ \\
\hline Alcohol 6\% & $11.6 \pm 0.2$ & $9.8 \pm 0.6$ & $6.6 \pm 0.7$ \\
\hline Alcohol 12\% & $10.4 \pm 0.6$ & $9.2 \pm 0.5$ & $7.0 \pm 0.6$ \\
\hline \multicolumn{4}{|c|}{$\begin{array}{l}\text { Parameters measured prior to ischaemia (pre-ischaemic), after } 5 \text { and } 30 \text { min of reperfusion. } \\
\text { Values are mean } \pm \text { standard error of mean }(n=6) \text {. LVDP=left ventricular developed pressure. } \\
* p<0.05 \text { v. control. }\end{array}$} \\
\hline
\end{tabular}

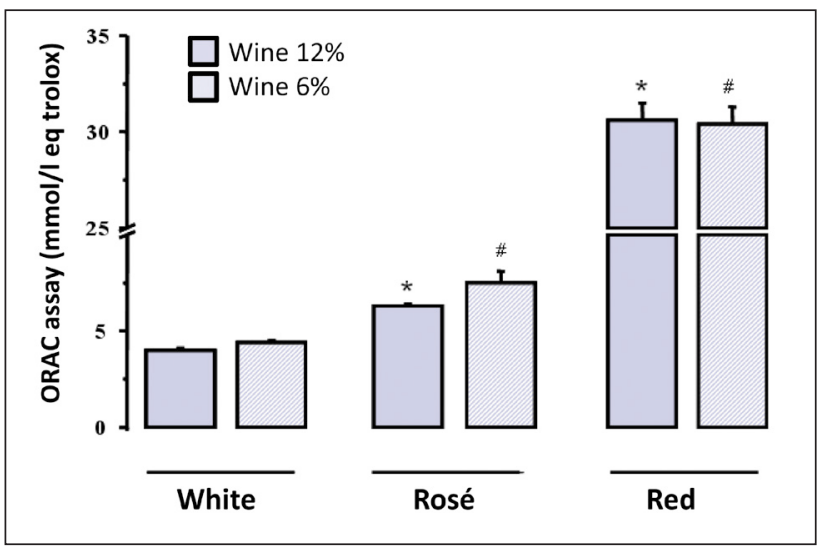

Fig. 1. Comparison of the antioxidant properties of white, rosé and red wines (12\% alcohol volume/volume) compared with the same wine after alcohol removal to a final concentration of $6 \%$. ${ }^{*} \mathrm{p}<0.05$ compared with white wine $12 \%$; ${ }^{*} \mathrm{p}<0.05$ compared with white wine $6 \% ; \mathrm{n}=6$ per group. ORAC = oxygen radical absorbance capacity.

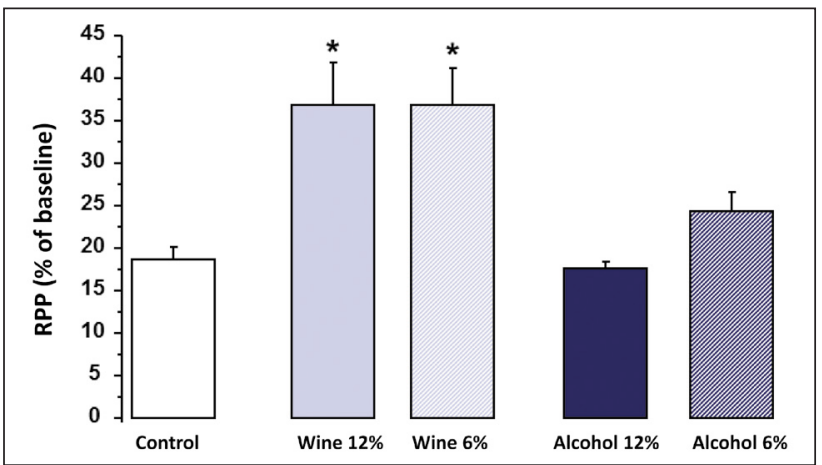

Fig. 2. Rate pressure product (RPP): heart rate $\mathrm{x}$ left ventricular developed pressure expressed as a percentage of baseline in isolated rat heart subjected to an ischaemia/reperfusion insult. ${ }^{*} \mathrm{p}<0.05$ v. control; $\mathrm{n}=6$ per group.

alcohol content in wine from $12 \%$ to $6 \%$ does not alter its antioxidant properties in vitro or its cardioprotective effect against I/R injury.

\section{Low-alcohol wine and antioxidant properties}

Our work clearly demonstrates that lirisation does not affect the antioxidant properties of the wine. Additional studies (data not shown) demonstrate that a complete removal of alcohol content does not alter the antioxidant properties of the wine, suggesting that these properties can be attributed mainly to components of the wine other than alcohol. Furthermore, our data show that the antioxidant capacity of red wine is superior to that of white wine. Other antioxidants including the polyphenols resveratrol and anthocyanins are found mainly in red wine. ${ }^{7}$ Further work will examine the contribution of each component in the antioxidant repertoire of wines.

\section{Low-alcohol wine and cardioprotective effect}

Low-dose ethanol drinking improves recovery of cardiac function and limits infarct size following an ischaemic insult. ${ }^{8}$ Non-alcoholic extracts of red wine and resveratrol also reduce infarct size. ${ }^{13}$ The fraction rich in polymeric proanthocyanidins separated from Argentinian red wine exerts the most powerful cardioprotective effect against ischaemia. ${ }^{14}$ None of these earlier studies, however, explored the cardioprotective effect of the wine against ischaemia in its entirety. The present study demonstrates that chronic and moderate administration of red wine with an alcohol content of $12 \%$ protected the rats against an ischaemic insult. Halving the alcohol 


\section{RESEARCH}

content did not alter this protective effect, suggesting that the alcohol fraction may play a minor role in cardioprotection relative to the polyphenols. Pretreatment of our animals with alcohol $6 \%$ or $12 \%$ alone, was not enough to protect the heart against an I/R insult. The cardioprotective effect of red wine is likely to be attributed to other antioxidants such as resveratrol that confer anti-ischaemic effects via the activation of prosurvival pathways, including the survivor activating factor enhancement (SAFE) pathway. ${ }^{15}$ It will be of interest to elucidate whether these other protective mechanisms still remain active when the alcohol content has been reduced to $6 \%$ and additional experiments, with complete removal of all alcohol content, will be required to establish if polyphenols alone can provide cardioprotection.

In conclusion, our findings suggest that the reduction of alcohol content to $6 \%$ in the wine did not alter its antioxidant and cardioprotective properties. Moderate and regular consumption of wines, with a lower content of alcohol than traditional wines, may confer a beneficial cardioprotective effect without the risks associated with higher alcohol consumption.

Acknowledgements. Part of this work was supported by the University of Cape Town (UCT), the National Research Foundation, the South African Medical Research Council (MRC) and Winetech. Kim Lamont was supported by the MRC and UCT. We would like to thank Le Boissonier Sarl for providing the wine.

\section{References}

1. Ronksley PE, Brien SE, Turner BJ, Mukamal KJ, Ghali WA. Association of alcohol consumption wit selected cardiovascular disease outcomes: a systematic review and meta-analysis. BMJ 2011;342:d671. [http://dx.doi.org/10.1136/bmj.d671]

2. Gordon T, Kannel WB. Drinking and mortality. The Framingham Study. Am J Epidemiol 1984;120:97107.

3. Fuchs CS, Stampfer MJ, Colditz GA, et al. Alcohol consumption and mortality among women. N Engl J Med 1995;332:1245-1250.

4. Bryson CL, Mukamal KJ, Mittleman MA, et al. The association of alcohol consumption and incident heart failure: the Cardiovascular Health Study. J Am Coll Cardiol 2006;48:305-311. [PMID: 16843180] Di Castelnuovo A, Costanzo S, Bagnardi V, Donati MB, Iacoviello L, de Gaetano G. Alcohol dosing and total mortality in men and women: an updated meta-analysis of 34 prospective studies. Arch Intern total mortality in men and women: an updated meta-analysis of 34 prospectin

6. Corrao G, Bagnardi V, Zambon A, La Vecchia C. A meta-analysis of alcohol consumption and the risk 6. Corrao G, Bagnardi V, Zambon A, La Vecchir
of 15 diseases. Prev Med 2004;38:613-619.

of 15 diseases. Prev Med 2004;38:613-619.
7. Lecour S, Lamont KT. Natural polyphenols and cardioprotection. Mini Rev Med Chem 2011;11:11911199 .

8. Opie LH, Lecour S. The red wine hypothesis: from concepts to protective signalling molecules. Eur Heart J 2007;28:1683-1693. [http://dx.doi.org/10.1093/eurheartj/ehm149]

9. Michael Paetzold. http://www.michaelpaetzold.com (accessed February 2012).

0. Blackhurst D, Wolmarans K, Marais AD. Wine dilates the brachial artery but does not increase the flow-mediated dilatation over two hours. S Afr J Enol Vitic 2007;28:11-16

11. Wollny T, Chabielska E, Malinowska-Zaprzalka M, Nazarko J, Rozmyslowicz-Szerminska W, Buczko W. Effects of Bulgarian red and white wines on primary hemostasis and experimental thrombosis in rats. Pol J Pharmacol 2003;55:1089-1096

12. Lecour S, Smith RM, Woodward B, Opie LH, Rochette L, Sack MN. Identification of a novel role for sphingolipid signaling in TNF alpha and ischemic preconditioning mediated cardioprotection. J Mol Cell Cardiol 2002:34:509-518

13. Sato M, Ray PS, Maulik G, et al. Myocardial protection with red wine extract. J Cardiovasc Pharmacol 2000;35:263-268.

14. Fantinelli JC, Schinella G, Cingolani HE, Mosca SM. Effects of different fractions of a red wine non-alcoholic extract on ischemia-reperfusion injury. Life Sci 2005;76:2721-2733. [http://dx.do org/10.1016/.1.lfs.2004.10.044

15. Lamont KT, Somers S, Lacerda L, Opie LH, Lecour S. Is red wine a SAFE sip away from cardioprotection? Mechanisms involved in resveratrol- and melatonin-induced cardioprotection. Pineal Res 2011;50:374-380. [http://dx.doi.org/10.1111/j.1600-079X.2010.00853.x]

Accepted 14 February 2012. 